\title{
INTERVENÇÕES EDUCATIVAS NO CONTEXTO DA HIPERTENSÃO ARTERIAL SISTÊMICA: UMA REVISÃO SISTEMÁTICA
}

\author{
INTERVENCIONES EDUCATIVAS EN EL CONTEXTO DE LA HIPERTENSIÓN \\ ARTERIAL SISTÉMICA: UNA REVISIÓN SISTEMÁTICA
}

\author{
EDUCATIONAL INTERVENTIONS IN THE CONTEXT OF SYSTEMIC ARTERIAL \\ HYPERTENSION: A SYSTEMATIC REVIEW
}

\author{
Elton Junior Siqueira GAMA ${ }^{1}$ \\ Walter Gonçalves de Queiroz JÚNIOR ${ }^{2}$ \\ Amanda Karine Barros Ferreira RODRIGUES ${ }^{3}$
}

RESUMO: A hipertensão arterial sistêmica é uma das maiores causas de morbidade no mundo, considerada um dos principais fatores de risco para desenvolvimento de doenças cardiovasculares. $\mathrm{O}$ presente trabalho tem como objetivo revisar sistematicamente as intervenções educativas desenvolvidas em serviços de saúde e na comunidade e sua atuação na prevenção e controle da hipertensão. Para tal, realizou-se uma revisão sistemática nas bases de dados LILACS, MEDLINE e SciELO, considerando trabalhos sem restrição de tempo e idioma. Os trabalhos que apresentaram resultados mais satisfatórios utilizaram estratégias mais interativas com uso de compartilhamento de experiências, participação ativa e educação baseada no modelo de assistência e parceria. Intervenções educacionais podem ampliar o conhecimento sobre a hipertensão arterial sistêmica, ajudar na adesão ao tratamento, proporcionar melhorias ligadas às condições clínicas do paciente e reduzir os níveis de pressão arterial.

PALAVRAS-CHAVE: Hipertensão arterial. Educação em saúde. Revisão sistemática.

RESUMEN: La hipertensión arterial sistémica es una de las más grandes causas de morbilidad en el mundo, considerada uno de los factores de riesgo para el desarrollo de enfermedades cardiovasculares. Este trabajo tiene como objetivo repasar sistemáticamente las intervenciones educativas desarrolladas en servicios de salud y en la comunidad y su actuación en la prevención y control de la hipertensión. Para eso, fue hecho un repaso sistemático en las bases de dados LILACS, MEDLINE y SciELO, considerando trabajos sin restricción de tiempo e idioma. Los trabajos que presentaban resultados más satisfactorios utilizaron estrategias más interactivas por medio de experiencias compartidas, participación activa y educación basada en el modelo de asistencia y trabajo en conjunto. Intervenciones

\footnotetext{
1 Universidade Federal de Alagoas (UFAL), Arapiraca - AL - Brasil. Complexo de Ciências Médicas e Enfermagem. Graduando de Medicina. ORCID: https://orcid.org/0000-0001-7052-3091. E-mail: elton.gama@arapiraca.ufal.br

${ }^{2}$ Universidade Federal de Alagoas (UFAL), Arapiraca - AL - Brasil. Complexo de Ciências Médicas e Enfermagem. Graduando de Medicina. ORCID: https://orcid.org/0000-0001-8436-1214. E-mail: walter.junior@arapiraca.ufal.br

${ }^{3}$ Universidade Federal de Alagoas (UFAL), Arapiraca - AL - Brasil. Complexo de Ciências Médicas e Enfermagem. Doutora em Ciências da Saúde. ORCID: https://orcid.org/0000-0002-6668-2820. E-mail: amanda.barros@arapiraca.ufal.br
} 
educacionales pueden ampliar el conocimiento sobre la hipertensión arterial sistémica, ayudar en la adhesión del tratamiento, proporcionar mejorías atribuidas a las condiciones clínicas del paciente y reducir los niveles de presión arterial.

PALABRAS CLAVE: Hipertención arterial. Educación para la salud Revisión sistemática.

ABSTRACT: Systemic arterial hypertension is one of the biggest causes of morbidity in the world and is considered one of the main risk factors for the development of cardiovascular diseases. This paper aims to systematically review the educational interventions developed in health services and in the community and their role in preventing and controlling hypertension. For that, a systematic review was carried out in the LILACS, MEDLINE and SciELO databases, considering works without time and language restrictions. The interventions that showed the most satisfactory results used more interactive strategies with the use of sharing experiences, active participation and education based on the assistance and partnership model. Educational interventions can increase knowledge about hypertension, help with treatment adherence, provide improvements related to the patient's clinical conditions and reduce blood pressure levels.

KEYWORDS: Hypertension. Health education. Systematic review.

\section{Introdução}

A Hipertensão Arterial Sistêmica (HAS) é uma das principais causas de morbidade no mundo, sendo uma condição clínica multifatorial caracterizada por níveis pressóricos suficientemente altos para aumentar o risco de surgimento de doenças cardiovasculares (DCVs), como: Infarto Agudo do Miocárdio (IAM), Insuficiência Cardíaca Congestiva (ICC), Acidente Vascular Encefálico (AVE), insuficiência renal e doença arterial periférica (KASPER et al., 2017).

De acordo com a Organização Mundial da Saúde (OMS), mais de um bilhão de pessoas em todo o mundo são hipertensas. No Brasil, a HAS atinge 32,5\% (36 milhões) de indivíduos adultos, mais de $60 \%$ dos idosos, contribuindo direta ou indiretamente para $50 \%$ das mortes por doenças cardiovasculares (SCALA et al., 2015). Além disso, a alta prevalência de HAS e consequentemente DCVs gera impactos econômicos e sociais intensos, devido aos custos de internação e sofrimento psicológico individual e familiar.

A $7^{\text {a }}$ Diretriz Brasileira de Hipertensão Arterial (2016) aponta que os principais fatores de risco para o desenvolvimento da HAS são: idade, sexo e etnia, excesso de peso e obesidade, ingesta de sal, ingestão de álcool, sedentarismo e fatores socioeconômicos. De modo que a grande maioria desses fatores de risco pode ser modificável através da educação em saúde, que consiste em um conjunto de práticas que fornecem aos indivíduos 
conhecimento em saúde suficiente para gerar promoção e prevenção de doenças. Nesse cenário, intensificar o acesso dos indivíduos à informação por meio de intervenções educativas em saúde pode colaborar para a disseminação de conhecimento em relação aos fatores de riscos e controle da HAS e contribuir com a mudança de comportamento.

Por conseguinte, o presente estudo apresenta como objetivo principal realizar uma revisão sistemática da literatura acerca das ações de educação em saúde no contexto da hipertensão arterial sistêmica, com o intuito de verificar se a educação em saúde é capaz de reduzir os fatores de risco associado à HAS, ao mesmo tempo observar quais medidas são mais efetivas nesse aspecto por meio da utilização de ferramenta que avalia a qualidade dos estudos e verificar a participação das ações na melhoria de desfechos comportamentais e clínicos.

\section{Método}

Como estratégia metodológica foi realizada uma revisão sistemática nas seguintes bases de dados: Literatura Latino-Americana e do Caribe em Ciências da Saúde (LILACS), Literatura Internacional em Ciências da Saúde (MEDLINE) e Scientific Electronic Library Online (SciELO). A busca ocorreu durante o mês de março de 2020, considerando trabalhos sem restrição de tempo e idiomas.

Foram utilizadas combinações com os seguintes termos presentes no Medical Subject Headings (MeSH) e seus correspondentes no Descritores em Ciências da Saúde (DeCS): (("health education"[MeSH Terms] OR "health promotion"[MeSH Terms]) AND "hypertension/prevention and control"[MeSH Terms]) AND ((("intervention studies"[All Fields] OR "clinical trial"[Publication Type]) OR "evaluation study"[Publication Type]) OR "program evaluation"[MeSH Terms]). Para as buscas no LILACS e SciELO também foram considerados os descritores em português.

Foram incluídos nesta revisão: (i) estudos de intervenção, podendo estes possuírem ou não randomização, com ou sem grupo controle, incluindo público de qualquer faixa etária; (ii) intervenções/ações educativas que avaliam desfechos clínicos e/ou comportamentais; (iii) estudos em que as intervenções educativas são realizadas e/ou lideradas por profissionais de saúde ou agentes comunitários.

Foram excluídos do estudo: (i) artigos em duplicidade; (ii) teses e dissertações; (iii) estudos com amostras pequenas ( $\mathrm{n}<20$ nos grupos de intervenção ou controle); (iv) estudos que não enfoquem em intervenções educativas voltadas à HAS; (v) trabalhos com 
intervenções educativas não claras; (vi) estudos em que o objetivo central é avaliação da experiência e conhecimento dos profissionais de saúde ou estudantes; (vii) estudos que foquem apenas na conscientização ou aquisição de conhecimento sem avaliar mudanças clínicas ou comportamentais; (viii) estudos multifacetados que não tem a intervenção educativa como a principal ação; (ix) estudos incluindo pacientes com comorbidades graves e hospitalizados.

A seleção dos artigos se deu pela leitura dos títulos e resumos, realizada de forma independente por dois dos autores. Após a leitura na íntegra, avaliação e seleção dos trabalhos, foram extraídas as seguintes informações dos estudos, com base no trabalho de Menegaz et al. (2018): autor, $n$ amostral, local de realização do estudo (países desenvolvidos ou em desenvolvimento), tipo de estudo (ensaio randomizado e controlado ou antes e depois), tipo de serviço de saúde (centro de saúde de atenção primária, hospitais, clínica médica, escolas), público-alvo, tipo de recurso educativo ou estratégia utilizada, dose da intervenção (1 a 3, 4 a 12 contatos com o público-alvo), tempo máximo de avaliação em meses ( 2 a 6,7 a 12, 13 a 24) e tipo de desfecho (clínico, comportamental ou clínico e comportamental), além dos resultados obtidos nos estudos.

$\mathrm{Na}$ avaliação dos resultados foram considerados parâmetros comportamentais: ingestão de sal, uso de tabaco, etilismo, prática de atividades físicas e o conhecimento sobre hipertensão. Os dados clínicos avaliados consistiram na avaliação das medidas da pressão arterial (PA).

Em relação à qualidade dos estudos, os incluídos foram avaliados a partir de instrumento proposto por Downs e Black (1998) e modificado por Menegaz et al. (2018). O instrumento é composto por 26 questões relativas à qualidade das informações do artigo, validade externa, validade e poder estatístico, oferecendo pontuação que varia de zero a 27. Os estudos foram classificados de acordo com suas pontuações em: excelente ( 25 a 27), bom (20 a 24), razoável (15 a 19), ou pobre ou limitado (14 ou menos), conforme critérios utilizados pelos autores.

\section{Resultados e discussão}

A busca dos textos selecionou 385 estudos em bases de dados indexadas, de modo que 220 presentes na Medline, 131 na SciELO e 34 na Lilacs. Não foram encontrados outros artigos em bases de dados distintas das citadas ou sem indexação de qualquer base de dados 
(literatura cinzenta). Também não foram encontradas duplicações, o que determinou a avaliação completa de 385 pesquisas para rastreamento, que terminou por excluir 359 estudos.

Na sequência, dos 26 artigos restantes lidos na íntegra, 13 foram excluídos devido: a intervenção educativa não estar clara (7 estudos), não serem aplicados por profissionais da saúde ( 2 estudos), apresentar amostra pequena ( 1 estudo), resultados parciais ( 1 estudo) e intervenções multifacetadas (1 estudo).

Desse modo, foram incluídos 13 artigos para avaliação qualitativa, de tal maneira que a revisão das referências dos textos incluídos não apontou nenhuma nova adição, o processo de escolha dos textos está indicado na figura 1.

Figura 1. Fluxograma de eleição dos artigos.

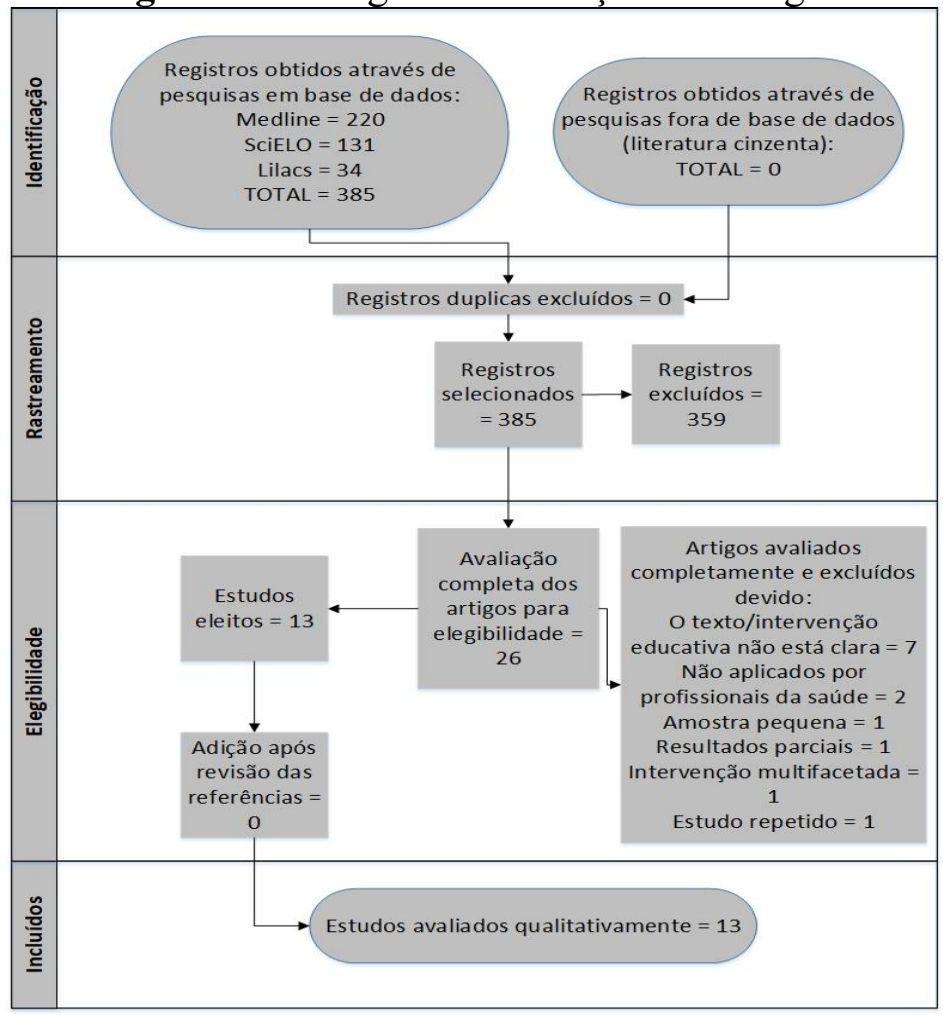

Fonte: Elaborado pelos autores

Em relação às características dos textos selecionados, a maioria ocorreu em países em desenvolvimento (MACHADO et al., 2016; JAFAR et al., 2010; LU et al., 2015; NGUYEN et al., 2018; OLIVEIRA et al., 2013; MOHAMMADI et al., 2006; KISIOGLU et al., 2004; MARTÍN et al., 2009) e é do tipo randomizado, havendo apenas 2 textos do tipo antes e depois (SMITH, MERRITT, PATEL 1997; NGUYEN et al., 2018). Os centros de saúde comunitário (MACHADO et al., 2016; LU et al., 2015; MOHAMMADI et al., 2006; KISIOGLU et al., 2004; MARTÍN et al., 2009) e a própria comunidade (SMITH, MERRITT, PATEL 1997; ISO et al., 1996; JAFAR et al., 2010; NGUYEN et al., 2018; OLIVEIRA et 
al., 2013) foram os locais em que mais ocorreram as intervenções, cada um com 5 estudos, embora hospitais/clínicas (BALCAZAR et al., 2009; MORI et al., 2010) e ambulatórios (FERRARA et al., 2012) também tenham sido utilizados.

O público-alvo de todos os estudos incluía adultos. Em 2 trabalhos (ISO et al., 1996; LU et al., 2015), além dos adultos, foi considerada a população idosa e um dos textos envolveu crianças (JAFAR et al., 2010). As estratégias metodológicas utilizadas foram: reuniões/palestras educacionais com 6 estudos (FERRARA et al., 2012; ISO et al., 1996; MARTIN et al., 2009; MOHAMMADI et al., 2006; MORI et al., 2010; SMITH, MERRITT, PATEL 1997), distribuição de material educativo (BALCAZAR et al., 2009), visitas domiciliares (JAFAR et al., 2010) e treinamento para autocuidado (KISIOGLU et al., 2004), algumas pesquisas utilizaram combinações dessas estratégias.

Diversos profissionais da saúde participaram dos estudos e o número de contatos entre os pesquisadores e os participantes sempre foi maior que três. A maior parte dos trabalhos acompanhou o público da pesquisa por mais de 1 ano (ISO et al., 1996; JAFAR, et al. 2010; LU et al., 2015; MARTÍN et al., 2009; MOHAMMADI et al., 2006; SMITH et al., 1997) e teve desfechos clínicos e comportamentais, com 4 estudos apresentando apenas desfechos clínicos (SMITH, MERRITT, PATEL 1997; JAFAR et al., 2010; MORI et al., 2010; MARTÍN et al., 2009). As principais características dos estudos e seus principais resultados estão apresentados no quadro 1.

Quadro 1. Análise qualitativa dos estudos incluídos

Autor: Balcazar et al. (2009).

Público-alvo: 98 adultos portadores de HAS sem atendimento médico regular.

Estudo e descrição da ação educativa: Estudo randomizado de 9 semanas. Grupo de intervenção: 58 participantes semanalmente receberam quatro módulos educacionais e chamadas telefônicas para acompanhamento. Grupo controle: 40 participantes.

Semanalmente receberam materiais educativos de forma geral.

Principais resultados: $O$ grupo de intervenção apresentou redução no consumo de sal e leve diminuição nos níveis pressóricos.

Autor: Ferrara et al. (2012)

Público-alvo: 188 hipertensos e em tratamento regular há pelo menos 6 meses

Estudo e descrição da ação educativa: Estudo randomizado com 1 ano de duração.

Grupo de intervenção: 94 participantes receberam consultas ambulatoriais e três reuniões mensais em pequenos grupos para aprimorar o conhecimento sobre a HAS. Grupo controle: 94 participantes receberam apenas consultas ambulatoriais.

Principais resultados: As médias de PA sistólica diminuíram no grupo de intervenção. Os participantes do grupo de intervenção também realizaram mais atividade física. 
Autor: Iso et al. (1996)

Público-alvo: 111 indivíduos entre 35 e 69 anos de idade portadores de HAS.

Estudo e descrição da ação educativa: Estudo randomizado com 1,5 ano de acompanhamento. Grupo de intervenção: acompanhou palestras e atividades de controle da hipertensão e prevenção do AVC três vezes durante os 6 primeiros meses e mais quatro vezes durante o restante do estudo. Grupo controle: acompanhou uma palestra semelhante ao do grupo de intervenção após 8 meses da randomização.

Principais resultados: No grupo de intervenção o consumo de sal diminuiu e a realização de atividade física aumentou

Autor: Jafar et al. (2010)

Público-alvo: 4023 indivíduos entre 5 e 39 anos de idade.

Estudo e descrição da ação educativa: Estudo randomizado com 2 anos de duração.

Grupo de intervenção: recebeu sessões domiciliares de disseminação de conceitos relacionados à prevenção e controle da HAS através da visita de agentes comunitários de saúde. Grupo de controle: não recebeu intervenção educativa.

Principais resultados: Redução dos níveis de PAS e PAD no grupo de intervenção.

Autor: Kisioglu et al. (2004)

Público-alvo: 400 mulheres entre 20-50 anos de idade escolhidas aleatoriamente.

Estudo e descrição da ação: Estudo randomizado com 6 meses de duração. Grupo de intervenção: 200 participantes tiveram treinamento em controle da PA e redução da obesidade. Grupo controle: 200 participantes. Não recebeu intervenção educativa.

Principais resultados: $\mathrm{O}$ grupo de intervenção apresentou redução dos níveis de PA. Também houve redução do sedentarismo e da ingestão de sal.

Autor: Lu et al. (2015)

Público-alvo: 360 portadores de HAS entre 40-75 anos de idade em tratamento.

Estudo e descrição da ação: Estudo randomizado com 2 anos de duração, de modo que os participantes foram divididos em três grupos:

Grupo 1: 120 participantes receberam material de leitura educativo.

Grupo 2: 120 participantes participaram de palestras mensais.

Grupo 3: 120 participantes participaram de palestras interativas mensais.

Principais resultados: Os três grupos apresentaram aumento do conhecimento e controle da HAS, aumento da prática de atividades físicas e diminuição dos fatores de risco relacionados à doença.

Autor: Machado et al. (2016)

Público-alvo: 212 indivíduos com hipertensão participantes do HIPERDIA.

Estudo e descrição da ação: Estudo randomizado com 1 ano de duração, que dividiu os participantes nos seguintes grupos:

Grupo 1: oficinas mensais para indivíduos com mais de 8 participações nas reuniões.

Grupo 2: oficinas mensais e visitas domiciliares.

Grupo 3: oficinas mensais para indivíduos com menos de 8 participações nas reuniões. As oficinas e palestras se utilizavam de metodologias ativas para fornecer educação e prevenção de doenças com ênfase na HAS.

Principais resultados: Os indivíduos de todos os grupos tiveram aumento da adesão ao tratamento não farmacológico da HAS bem como diminuíram os fatores de risco relacionados à doença, exemplo disso foi a diminuição no consumo de sal. 
Autor: Martín et al. (2009)

Público-alvo: 2180 portadores de HAS escolhidos de forma aleatória.

Estudo e descrição da ação: Estudo randomizado durante 1 ano. Grupo de intervenção: Realizou-se de forma ativa 3 sessões, 1 workshop e 1 sessão de reforço. Grupo controle: Não recebeu intervenção educativa.

Principais resultados: Diminuição da ingestão de sal e do risco cardiovascular no grupo de intervenção.

Autor: Mohammadi et al. (2006)

Público-alvo: 150 portadores de HAS escolhidos de forma aleatória.

Estudo e descrição da ação: Estudo randomizado com 1,5 anos de duração. Grupo de intervenção: 75 pacientes frequentaram 4 sessões de conhecimento sobre a HAS e 11 sessões mensais que consistiam em troca de informações sobre o autocuidado, a partir de um modelo de assistência e parceria. Grupo controle: 75 receberam cuidados rotineiros, sem medidas de educação em saúde.

Principais resultados: Diminuição dos níveis de PAS e PAD com aumento da taxa de controle da doença entre o grupo de intervenção.

Autor: Mori et al. (2010)

Público-alvo: 75 indivíduos hipertensos de um centro de saúde.

Estudo e descrição da ação: Estudo randomizado com 9 meses de duração. Grupo de intervenção: 41 participantes receberam palestras e orientação farmacêutica para controle da HAS. Grupo controle: 34 participantes não receberam a intervenção.

Principais resultados: Os participantes do grupo de intervenção apresentaram maior redução da PA comparado ao grupo controle.

Autor: Nguyen et al. (2018)

Público-alvo: 157 indivíduos com hipertensão mal controlada.

Estudo e descrição da ação: Estudo randomizado com 1 ano de duração. Grupo de intervenção: 78 indivíduos participaram de duas estratégias: troca de experiências ("contar histórias") e distribuição de material educativo (DVDs) referente ao controle de fatores de risco relacionados à HAS. Grupo controle: 79 participantes não receberam a intervenção.

Público-alvo: Ambas as estratégias realizadas no grupo de intervenção foram capazes de diminuir os níveis de PA e aumentar o controle da HAS.

Autor: Oliveira et al. (2013)

Público-alvo: 216 portadores adultos de HAS.

Estudo e descrição da ação: Estudo antes e depois com 6 meses de duração. Consistia na realização de troca de experiências, fornecimento de material educativo e avaliação do cuidado.

Principais resultados: Cresceu a adesão ao tratamento não medicamentoso da HAS.

Autor: Smith et al. (1997)

Público-alvo: 97 indivíduos adultos membros de igrejas selecionadas.

Estudo e descrição da ação: Estudo antes e depois com 2 anos de duração. Usou sessões/ palestras para a disseminação de conhecimentos sobre a HAS e seus fatores de risco com ênfase na redução dos níveis de sódio e colesterol.

Principais resultados: Os participantes tiveram aumento do conhecimento sobre hipertensão e redução dos níveis da PAS e PAD. 
A orientação adequada aos indivíduos em relação ao consumo de sal pode ter impacto sobre os níveis pressóricos destes. Esse fato foi analisado por alguns autores (BALCAZAR et al., 2009; MACHADO et al., 2016; ISO et al., 1996; MARTIN et al., 2009; KISOGLU et al., 2014), que utilizando-se de sessões, palestras e distribuição de material didático conseguiram estabelecer uma correlação entre educação em saúde sobre a HAS e consumo de sal.

Esses estudos que acompanharam os participantes por 2-18 meses em sua totalidade determinaram que a educação em saúde, por meio das metodologias citadas, foi capaz de reduzir os níveis de PA dos participantes.

Outrossim, a educação em saúde também foi capaz de diminuir o sedentarismo dos participantes (LU et al., 2015; OLIVEIRA et al., 2013; KISIOGLU et al., 2004; FERRARA et al., 2012). Demonstrou-se que por meio de reuniões, trocas de experiências entre os hipertensos e distribuição de material didático acerca da HAS por 6 meses a 2 anos, o nível de participação em atividades físicas entre os participantes aumentou.

Esse fato é benéfico sobre a saúde dos indivíduos pois os exercícios físicos promovem cardioproteção e consequentemente reduzem as chances de isquemia do miocárdio, logo a possibilidade de ocorrência de agravos cardiovasculares adversos pode estar reduzida (BORGES; LESSA, 2015).

Outro impacto positivo da educação em saúde foi acerca do conhecimento da HAS em pacientes hipertensos, os estudos (SMITH, MERRITT, PATEL 1997; LU et al., 2015; MORI et al., 2010) que avaliaram essa correlação se utilizaram de questionários aplicados antes e após as intervenções, de modo que todos eles demonstraram aumento do conhecimento dos participantes sobre a doença.

Nesse aspecto, o entendimento do processo saúde-doença tem impactos sobre os indivíduos ao melhorar a adesão à terapêutica e consequentemente a expectativa de vida (CARVALHO et al., 2013). Deve-se levar em conta também, que os estudos demonstraram que a escolaridade dos participantes influencia na compreensão dos aspectos abordados, potencializando a aprendizagem.

Por outro lado, os estudos analisados (LU et al., 2015; OLIVEIRA et al., 2013) não conseguiram estabelecer resultados significativos da relação entre a educação em saúde e o consumo de álcool e tabaco durante o período de avaliação de 6-24 meses, através da realização de reuniões e distribuição de material didático referente a HAS. Esse fato pode ser explicado pela capacidade do álcool e cigarro em gerar dependência nos seus usuários, dificultando as estratégias de encerramento do vício. 
Em relação aos níveis de PA, os treze trabalhos verificaram algum tipo de alteração, seja na média das pressões sistólicas e diastólicas ou no número de pacientes que conseguiram controlar a PA. Cinco trabalhos apresentaram redução significativa da PA após a intervenção (FERRARA et al., 2012; LU et al., 2015; MOHAMMADI et al., 2006; OLIVEIRA et al., 2013; SMITH, MERRITT, PATEL 1997), como pode ser observado na figura 2.

Figura 2. Tabela das medidas de pressão arterial nos grupos (continua)

\begin{tabular}{|c|c|c|c|c|}
\hline Autor (ano) & Grupo(s) & $\begin{array}{l}\text { Valores pré- } \\
\text { intervenção }\end{array}$ & $\begin{array}{l}\text { Valores pós- } \\
\text { intervenção }\end{array}$ & $\begin{array}{l}\mathbf{p} \\
\text { (antes/depois) }\end{array}$ \\
\hline \multicolumn{5}{|c|}{ PAS e PAD, mmHg ( $\pm \mathrm{DP})$} \\
\hline \multirow[t]{2}{*}{$\begin{array}{l}\text { Balcazar et } \\
\text { al. }(2009)\end{array}$} & I & $\begin{array}{l}22 \% \text { dos sujeitos } \\
\text { tinham PA } \\
<120 / 80\end{array}$ & $\begin{array}{l}26 \% \text { dos sujeitos } \\
\text { apresentavam } \mathrm{PA}< \\
120 / 80\end{array}$ & na \\
\hline & C & $\begin{array}{l}18 \% \\
\text { apresentatam } \\
\mathrm{PA}<120 / 80\end{array}$ & $\begin{array}{l}15 \% \text { apresentavam } \mathrm{PA}< \\
120 / 80\end{array}$ & \\
\hline \multirow[t]{2}{*}{$\begin{array}{l}\text { Ferrara et al. } \\
(2012)\end{array}$} & $\begin{array}{l}\text { I (PAS) } \\
C \text { (PAS) }\end{array}$ & $\begin{array}{l}136,0=17 \\
132,3 \pm 15\end{array}$ & $\begin{array}{l}124,5 \pm 10 \\
133,5 \pm 15 \\
\mathrm{p}^{\mathrm{d}} 0,001\end{array}$ & $\begin{array}{l}0.001 \\
\mathrm{n} 5\end{array}$ \\
\hline & $\begin{array}{l}I(P A D) \\
C(P A D)\end{array}$ & $\begin{array}{l}85,4 \pm 12 \\
83,3 \pm 9\end{array}$ & $\begin{array}{l}77,9=9 \\
81,3=9 \\
p^{4} 0.01\end{array}$ & $\begin{array}{l}0,001 \\
\mathrm{n} 5\end{array}$ \\
\hline \multirow[t]{2}{*}{$\begin{array}{l}\text { Iso et al } \\
(2010)\end{array}$} & $\begin{array}{l}\text { I (PAS) } \\
C \text { (PAS) }\end{array}$ & $\begin{array}{l}149,5=8,0 \\
148,4=10,1 \\
\mathrm{p}^{2} 0.26\end{array}$ & $\begin{array}{l}136,3 \pm 13,2 \\
141,0 \pm 16,0 \\
p^{d} 0.04\end{array}$ & \\
\hline & $\begin{array}{l}I(\mathrm{PAD}) \\
\mathrm{C}(\mathrm{PAD})\end{array}$ & $\begin{array}{l}84,1 \pm 7,6 \\
83,3 \pm 8,4 \\
p^{2} 0,30\end{array}$ & $\begin{array}{l}79,0=9,0 \\
78,6=9,6 \\
\mathrm{p}^{\mathrm{d}} 0,41\end{array}$ & na \\
\hline \multirow[t]{2}{*}{$\begin{array}{l}\text { Jafar et al } \\
(2010)\end{array}$} & $\begin{array}{l}\text { I (PAS) } \\
\mathrm{C} \text { (PAS) }\end{array}$ & $\begin{array}{l}114,0=14 \\
115,0=14\end{array}$ & $\begin{array}{l}\text { Aumento de } 0,1 \\
\text { Aumento de } 1,5 \\
\mathrm{p}^{\mathrm{d} 0.02}\end{array}$ & \\
\hline & $\begin{array}{l}I(P A D) \\
C(P A D)\end{array}$ & $\begin{array}{l}74,0 \pm 11 \\
74,0 \pm 11\end{array}$ & $\begin{array}{l}\text { Aumento de } 0,6 \\
\text { Aumento de } 2,1 \\
p^{d} 0,002\end{array}$ & $\mathrm{n}$ \\
\hline \multirow[t]{2}{*}{$\begin{array}{l}\text { Kisioglu et al. } \\
\text { (2004) }\end{array}$} & I & $\begin{array}{l}31,5 \% \text { tinham } \\
\text { pressão > }>130 / 85\end{array}$ & $\begin{array}{l}21,5 \% \text { tinham } \mathrm{PA}> \\
130 / 85\end{array}$ & \\
\hline & C & $\begin{array}{l}31 \% \text { tinham } \\
\text { pressão }>130 / 85 \\
\mathrm{p}^{2} 0,964\end{array}$ & $\begin{array}{l}28,5 \% \text { tinham } P A> \\
130 / 85 \\
p^{=0,310}\end{array}$ & na \\
\hline \multirow[t]{2}{*}{$\begin{array}{l}\text { Lu et al. } \\
(2015)\end{array}$} & $\begin{array}{l}1 \text { (PAS) } \\
2 \text { (PAS) } \\
3 \text { (PAS) }\end{array}$ & $\begin{array}{l}140,2=17,9 \\
143,9=16,2 \\
148,7=21,5 \\
p^{2} 0,003\end{array}$ & $\begin{array}{l}139,4 \pm 16,7 \\
134,8 \pm 15,9 \\
133,7 \pm 13,6 \\
p^{d} 0.014\end{array}$ & $\begin{array}{l}0,611 \\
<0,001 \\
<0,001\end{array}$ \\
\hline & $\begin{array}{l}1 \text { (PAD) } \\
2 \text { (PAD) } \\
3 \text { (PAD) }\end{array}$ & $\begin{array}{l}86,7 \pm 11,2 \\
85,8 \pm 10,7 \\
90,7 \pm 16,5 \\
p^{2} 0,010\end{array}$ & $\begin{array}{l}85,2=10,66 \\
80,4=11,2 \\
81,2=8,0 \\
\mathbf{p}^{d} 0,001\end{array}$ & $\begin{array}{l}0,177 \\
<0,001 \\
<0,001\end{array}$ \\
\hline
\end{tabular}




\begin{tabular}{|c|c|c|c|c|}
\hline Autor (ano) & Grupo(s) & $\begin{array}{l}\text { Valores pré- } \\
\text { intervenção }\end{array}$ & $\begin{array}{l}\text { Valores pós- } \\
\text { intervenção }\end{array}$ & $\begin{array}{l}\text { p } \\
\text { (antes/depois) }\end{array}$ \\
\hline \multicolumn{5}{|c|}{ PAS e PAD, mmHg ( $\pm D P$ ) } \\
\hline \multirow[t]{2}{*}{$\begin{array}{l}\text { Machado et } \\
\text { al. (2016) }\end{array}$} & $\begin{array}{l}1 \text { (PAS) } \\
2 \text { (PAS) } \\
3 \text { (PAS) }\end{array}$ & $\begin{array}{l}125,0=20 \\
117,0=14,8 \\
124,0=20,0\end{array}$ & $\begin{array}{l}121,0 \pm 18,5 \\
119,0 \pm 18,0 \\
122,0 \pm 16,9\end{array}$ & $\begin{array}{l}0,356 \\
0,839 \\
0,937\end{array}$ \\
\hline & $\begin{array}{l}1(\mathrm{PAD}) \\
2(\mathrm{PAD}) \\
3(\mathrm{PAD})\end{array}$ & $\begin{array}{l}72,2 \pm 11,8 \\
70,6 \pm 11,6 \\
74,7 \pm 16,0\end{array}$ & $\begin{array}{l}68,4=18,9 \\
69,5=16,0 \\
70,9=12,8\end{array}$ & $\begin{array}{l}0,749 \\
0,809 \\
0,300\end{array}$ \\
\hline \multirow[t]{2}{*}{$\begin{array}{l}\text { Martin et al. } \\
(2009)\end{array}$} & $\begin{array}{l}\text { I (PAS) } \\
\text { C (PAS) }\end{array}$ & $\begin{array}{l}137,9=19,6 \\
139,1=16,3\end{array}$ & $\begin{array}{l}132,6 \pm 19,6 \\
132,0 \pm 16,3\end{array}$ & \multirow{2}{*}{ na } \\
\hline & $\begin{array}{l}\text { I (PAD) } \\
C(P A D)\end{array}$ & $\begin{array}{l}83,8 \pm 10,8 \\
79,6 \pm 11,5\end{array}$ & $\begin{array}{l}79,9=10,8 \\
76,9=11,5\end{array}$ & \\
\hline \multirow[t]{2}{*}{$\begin{array}{l}\text { Mohammadi } \\
\text { et al (2006) }\end{array}$} & $\begin{array}{l}\text { I (PAS) } \\
C \text { (PAS) }\end{array}$ & $\begin{array}{l}175,5 \\
167,6\end{array}$ & $\begin{array}{l}144,7 \\
163,2\end{array}$ & 0,00 \\
\hline & $\begin{array}{l}\text { I (PAD) } \\
\text { C (PAD) }\end{array}$ & $\begin{array}{l}98,5 \\
99,5\end{array}$ & $\begin{array}{l}91,5 \\
98,4\end{array}$ & 0,05 \\
\hline \multirow[t]{2}{*}{$\begin{array}{l}\text { Mori et al. } \\
(2010)\end{array}$} & I & $\begin{array}{l}51 \% \\
\text { apresentaram, } \\
\text { PA > ou } \\
\text { próxima a } 140 \text { / } \\
90 .\end{array}$ & $\begin{array}{l}48 \% \text { dos que } \\
\text { apresentavam PA >ou } \\
\text { próxima a } 140 / 90, \\
\text { normalizaram a PA. }\end{array}$ & \multirow[b]{2}{*}{ na } \\
\hline & $\mathrm{C}$ & $\begin{array}{l}71 \% \\
\text { apresentaram } \\
\text { PA> ou } \\
\text { próxima a } 140 \text { / } \\
90 .\end{array}$ & $\begin{array}{l}21 \% \text { dos que } \\
\text { apresentaram PA >ou } \\
\text { próxima a } 140 / 90, \\
\text { normalizaram a PA. }\end{array}$ & \\
\hline \multirow[t]{2}{*}{$\begin{array}{l}\text { Nguyen et al. } \\
\text { (2018) }\end{array}$} & $\begin{array}{l}1 \text { (PAS) } \\
2 \text { (PAS) }\end{array}$ & $\begin{array}{l}150,4=17,5 \\
144,3=8,9\end{array}$ & $\begin{array}{l}139,7 \pm 16,7 \\
138,5 \pm 16,7\end{array}$ & \multirow{2}{*}{ na } \\
\hline & $\begin{array}{l}1 \text { (PAD) } \\
2 \text { (PAD) }\end{array}$ & $\begin{array}{l}91,3 \pm 9,3 \\
87,8 \pm 8,1\end{array}$ & $\begin{array}{l}84,7=9,8 \\
84,1=12,5\end{array}$ & \\
\hline $\begin{array}{l}\text { Oliveira el al. } \\
\text { (2013) }\end{array}$ & I & $\begin{array}{l}44,9 \text { \% estaram } \\
\text { com pressào } \\
<140 / 90\end{array}$ & $\begin{array}{l}76,6 \% \text { estaram com } \\
\text { pressào }<140 / 90\end{array}$ & 0,004 \\
\hline \multirow{2}{*}{$\begin{array}{l}\text { Smith el al } \\
\text { (1997) }\end{array}$} & I (PAS) & 147,0 & 140,0 & $<0.0000$ \\
\hline & $\mathrm{I}(\mathrm{PAD})$ & 85,0 & 83,0 & $<0,008$ \\
\hline
\end{tabular}

I: grupo de intervenção; C: grupo controle; 1, 2, 3: Intervenções diferentes em um mesmo trabalho; PAS: pressão arterial sistólica; PAD: pressão arterial diastólica; DP: desvio padrão; $p^{\mathrm{a}}$ : $\mathrm{p}$ da comparação dos grupos na linha de base; $\mathrm{p}^{\mathrm{d}}$ : p da comparação dos grupos após a intervenção; ns: Não significativo; na: Não apresentado.

Fonte: Elaborado pelos autores

Ferrara et al. (2012) evidenciaram redução da PAS e da PAD no grupo de intervenção. $\mathrm{Lu}$ et al. (2015) abordam que tanto a PA sistólica quanto a diastólica diminuíram significativamente e a proporção de PA normalizada aumentou significativamente após a intervenção nos grupos 2 e 3.

Em Jafar et al. (2010), pode ser percebido pequeno aumento na PAS e PAD, no entanto o aumento foi menor no grupo de intervenção, que consistiu em educação domiciliar, o que apresenta um efeito benéfico sobre a PA de crianças e jovens adultos. Também são 
percebidas mudanças significativas em: Mohammadi et al. (2006), Oliveira et al. (2013) e Smith et al. (1997).

Os trabalhos que apresentaram resultados mais satisfatórios em relação a diminuição e/ou controle da PA, tiveram um tempo de acompanhamento maior com os sujeitos do estudo, geralmente superior a 12 meses e utilizaram estratégias mais interativas com uso de compartilhamento de experiências, participação ativa e educação baseada no modelo de assistência e parceria.

No contexto da HAS, intervenções educacionais com participação ativa podem ampliar o conhecimento sobre a doença, ajudar na adesão ao tratamento regular e proporcionar melhorias ligadas ao controle da PA (LU et al., 2015).

Outro aspecto observado em relação às intervenções é o benefício em se trabalhar em pequenos grupos com trocas de experiência. A formação de grupos gera um potencial que favorece a ampliação das interações sociais e dinamismo, com compartilhamento de experiências e problemas comuns relacionados às condições de saúde do sujeito (CRABTREE et al., 2015).

Ferrara e colaboradores (2012) consideram que em termos de custo-benefício a abordagem de pequenos grupos para um programa educacional para melhorar os hábitos de vida pode ser considerada eficaz e menos cara, especialmente considerando a redução esperada de eventos cardiovasculares ao longo do tempo.

O modelo de educação voltado à assistência e parceria, também compõe o rol de estratégias interativas. Segundo esse modelo, a atenção deve ser dada não apenas às características epidemiológicas e fisiológicas da doença, mas também às características dos pacientes e às interações entre os sujeitos e profissionais no processo terapêutico.

A parceria aumenta o envolvimento, motivação e responsabilidade das pessoas. Mohammadi et al. (2006) evidenciam que esse tipo de intervenção foi eficaz na maioria dos índices de controle da hipertensão, como média da PA sistólica e diastólica, taxa de hipertensão controlada e diminuição dos fatores de risco cardiovasculares.

A avaliação da qualidade dos estudos está expressa na figura 3. A média total dos escores foi de 20,1 pontos ( $\mathrm{DP}=2,59)$ de acordo com o instrumento de Downs e Black, adaptado por Menegaz et al. (2018). A menor pontuação foi 15 e as maiores pontuações totalizaram 24 pontos.

Oito estudos foram classificados como bons e cinco foram considerados com qualidade razoável. Segundo os itens do instrumento, a avaliação identificou maiores problemas metodológicos relacionados a confusão e relato. 
Figura 3. Tabela da avaliação da qualidade das intervenções, de acordo com os critérios de Downs e Black

\begin{tabular}{|c|c|c|c|c|c|c|}
\hline Autores, ano & $\begin{array}{c}\text { Relato } \\
(0 \text { a 11) }\end{array}$ & $\begin{array}{c}\text { Validade } \\
\text { externa } \\
\left(\begin{array}{l}0 \\
\text { a } 3\end{array}\right)\end{array}$ & $\begin{array}{l}\text { Viés* } \\
(0 \text { a 6) }\end{array}$ & $\begin{array}{l}\text { Confundimento } \\
\left(\begin{array}{l}0 \text { a } 6)\end{array}\right.\end{array}$ & $\begin{array}{l}\text { Poder } \\
\left(\begin{array}{l}0 \text { a } 1)\end{array}\right.\end{array}$ & $\begin{array}{l}\text { Somatório } \\
\text { (0 a 27) }\end{array}$ \\
\hline Jafar et al. (2010) & 9 & 3 & 6 & 5 & 1 & 24 \\
\hline Lu et al. (2015) & 9 & 3 & 6 & 5 & 1 & 24 \\
\hline $\begin{array}{l}\text { Machado et al. } \\
\text { (2016) }\end{array}$ & 9 & 3 & 5 & 4 & 1 & 22 \\
\hline $\begin{array}{l}\text { Mohammadi et al. } \\
\text { (2006) }\end{array}$ & 8 & 3 & 5 & 5 & 1 & 22 \\
\hline $\begin{array}{l}\text { Nguyen et al. } \\
\text { (2018) }\end{array}$ & 8 & 3 & 5 & 5 & 1 & 22 \\
\hline Ferrara et al. (2012) & 7 & 3 & 5 & 5 & 1 & 21 \\
\hline $\begin{array}{l}\text { Balcazar et al. } \\
(2009)\end{array}$ & 8 & 3 & 4 & 4 & 1 & 20 \\
\hline $\begin{array}{l}\text { Kisioglu et al } \\
(2004)\end{array}$ & 7 & 3 & 5 & 4 & 1 & 20 \\
\hline Martin et al. (2009) & 7 & 3 & 4 & 4 & 1 & 19 \\
\hline Isso et al. (1996) & 7 & 3 & 4 & 3 & 1 & 18 \\
\hline Mori et al. (2010) & 5 & 3 & 5 & 4 & 1 & 18 \\
\hline $\begin{array}{l}\text { Oliveira et al. } \\
(2013)\end{array}$ & 7 & 2 & 4 & 3 & 1 & 17 \\
\hline Smith et al. (1997) & 7 & 1 & 5 & 2 & 0 & 15 \\
\hline Média (DP) & $\begin{array}{c}7,5 \\
(1,08)\end{array}$ & $2,7(0,57)$ & $\begin{array}{c}4,8(0,6 \\
6)\end{array}$ & $4,0(0,91)$ & $\begin{array}{c}0,9 \\
(0,26)\end{array}$ & $20,1(2,59)$ \\
\hline
\end{tabular}

* Questão 14 do instrumento Downs e Black foi excluída.

Fonte: Elaborado pelos autores

Observa-se que todos os estudos tiveram classificação razoável/bom, no que diz respeito à qualidade das evidências. Parte se deve a uma boa descrição das atividades de intervenção, com metodologias bem esclarecidas, além da representatividade das populações alvo e equipes envolvidas nos estudos. Os principais problemas na qualidade ocorreram por conta da ausência do relato dos principais fatores de confusão, ausência de dados sobre eventos adversos e limitações na tentativa de ocultar o tipo de intervenção para pacientes e equipes de saúde.

\section{Considerações finais}

Conclui-se que as intervenções educativas no contexto da HAS, principalmente quando reproduzidas de forma ativa, resultam em melhorias no comportamento que acabam levando a diminuição dos fatores de risco que influenciam no aumento da PA. Entre as estratégias adotadas, merecem atenção aquelas com maior tempo de acompanhamento, interação entre pequenos grupos e baseadas no modelo de assistência e parceria.

A presente revisão possibilita aos profissionais de saúde conseguir informações sobre estratégias de educação em saúde e oferece subsídios para possíveis adaptações e implementações junto à comunidade, possibilitando o incentivo à busca de novas ações. 
Reforçamos que ainda são necessários estudos futuros que abordem principalmente o uso contínuo das intervenções no âmbito das doenças crônicas e suas repercussões a longo prazo.

\section{REFERÊNCIAS}

BALCAZAR, H. G. et al. A randomized community intervention to improve hypertension control among mexican americans: using the promotoras de salud community outreach model. Journal of Health Care for the Poor and Underserved, v. 20, n. 4, p. 1079-1094, 2009.

BORGES, J. P.; LESSA, M. A. Mechanisms involved in exercise-induced cardioprotection: A systematic review. Arquivos Brasileiros de Cardiologia, v. 105, n. 1, p. 71-81, 2015.

CARVALHO, M. V. et al. The influence of hypertension on quality of life. Arquivos Brasileiros de Cardiologia, v. 100, n. 2, p. 164-174, 2013.

CRABTREE, K. et al. African American men's preferences for a community-based diabetes management program. The diabetes educator. 41(1): p. 118-126, 2015.

DOWNS, S.H.; BLACK, N. The feasibility of creating a checklist for the assessment of the methodological quality both of randomised and non-randomised studies of health care interventions. Journal of Epidemiology Community Health. 52(6): p. 377-84, 1998.

FERRARA, A. L. et al. Lifestyle educational program strongly increases compliance to nonpharmacologic intervention in hypertensive patients: a 2-year follow-up study. The Journal of clinical hypertension. vol. 14. Oct. 2012.

ISO, H. et al. Community-Based Education Classes for Hypertension Control. Journal of the American Heart Association, vol, 27. Apr. 1996.

JAFAR, T. H. et al. Community based lifestyle intervention for blood pressure reduction in children and young adults in developing country: Cluster randomised controlled trial. BMJ (Online), v. 340, n. 7762, p. 1-7, 2010.

KASPER, D. L. et al. Medicina interna de Harrison. 19 ed. Porto Alegre: AMGH Editora, 2017.

KISIOGLU, A. N. et al. Improving Control of High Blood Pressure among Middle-aged Turkish Women of Low Socio-economic Status through Public Health Training. Croatian Medical Journal, vol.45. 2004.

LU, C. H. et al. Community-based interventions in hypertensive patients: A comparison of three health education strategies. BMC Public Health, v. 15, n. 1, p. 1-9, 2015.

MACHADO, J. C. et al. Adherence to non-pharmacological treatment: Analysis of the impact of three health educational and nutritional strategies in hypertensive patients. Revista de Nutricao, v. 29, n. 1, p. 11-22, 2016. 
MARTÍN, C. R. et al. Eficacia de una intervención educativa grupal sobre cambios en los estilos de vida en hipertensos en atención primaria: un ensayo clínico aleatorio. Journal of Agricultural and Food Chemistry, v. 1, n. 2, p. 193, 1953.

MENEGAZ, A. M. et al. Intervenções educativas em serviços de saúde e saúde bucal: revisão sistemática. Revista de Saúde Pública, vol.52:52. 2018.

MOHAMMADI, E. et al. Evaluation of "partnership care model" in the control of hypertension. International Journal of Nursing Practice, v. 12, n. 3, p. 153-159, 2006.

MORI, A. L. P. M. et al. Pharmaceutic guidance to hypertensive patients at USP University Hospital: Effect on adherence to treatment. Brazilian Journal of Pharmaceutical Sciences, v. 46, n. 2, p. 353-362, 2010.

NGUYEN, H. L. et al. Culturally adaptive storytelling intervention versus didactic intervention to improve hypertension control in Vietnam- 12 month follow up results: A cluster randomized controlled feasibility trial. PLoS ONE, v. 13, n. 12, p. 1-13, 2018.

OLIVEIRA, T. L. et al. Eficácia da educação em saúde no tratamento não medicamentoso da hipertensão arterial. ACTA Paulista de Enfermagem, v. 26, n. 2, p. 179-184, 2013.

ORGANIZAÇÃO PAN-AMERICANA DA SAÚDE; ORGANIZAÇÃO MUNDIAL DE SAÚDE. Dia mundial da hipertensão. OPAS/OMS, 2016. Disponível em:

https://www.paho.org/bireme/index.php?option=com_content\&view=article\&id=330:diamundial-da-hipertensao-2016\&Itemid=183\&lang=pt. Acesso em: 20 mai. 2020.

SCALA, L. C.; MAGALHÃES, L. B.; MACHADO, A. Epidemiologia da hipertensão arterial sistêmica. In: Moreira SM, Paola AV; Sociedade Brasileira de Cardiologia. Livro Texto da Sociedade Brasileira de Cardiologia. 2a ${ }^{\text {a }}$ ed. São Paulo: Manole; 2015.

SMITH, E. D.; MERRITT, S. L.; PATEL, M. K. Church-based education: An outreach program for African Americans with hypertension. Ethnicity and Health, v. 2, n. 3, p. 243253, 1997.

SOCIEDADE BRASILEIRA DE CARDIOLOGIA. $7^{\text {a }}$ Diretriz Brasileira De Hipertensão Arterial Sistêmica. Arquivos Brasileiros de Cardiologia, v. 107, p. 5, 2016. 


\section{Como referenciar este artigo}

GAMA, E. J. S.; JÚNIOR, W. G. Q.; RODRIGUES, A. K. B. F. Intervenções educativas no contexto da Hipertensão Arterial Sistêmica: uma revisão sistemática. Temas em Educ. e Saúde, Araraquara, v. 17, n. 00, e021010, 2021. e-ISSN 2526-3471. DOI: https://doi.org/10.26673/tes.v17i00.14729

Submetido em: 02/02/2021

Revisões requeridas em: 16/03/2021

Aprovado em: 24/05/2021

Publicado em: 28/06/2021 